\title{
TTS: High-Speed Signatures on a Low-Cost Smart Card
}

\author{
Bo-Yin Yang ${ }^{1}$, Jiun-Ming Chen ${ }^{2}$, and Yen-Hung Chen ${ }^{3}$ \\ 1 Mathematics Department, Tamkang University, Tamsui, Taiwan, by@moscito.org \\ ${ }^{2}$ Chinese Data Security Inc. \& National Taiwan University, jmchen@math.ntu . edu.tw \\ ${ }^{3}$ Comp. Sci. \& Info. Eng., Nat'l Taiwan U., Taipei, Taiwan, r92014@csie.ntu. edu.tw
}

\begin{abstract}
TTS is a genre of multivariate digital signature schemes first proposed in 2002. Its public map is composed of two affine maps sandwiching a Tame Map, which is a map invertible through serial substitution and solving linear equations. We implement the signing and key generation operations for a TTS instance with 20-byte hashes and 28-byte signatures, on popular extant microcontroller cores compatible to the Intel 8051 . Our tests demonstrates that TTS can be even faster than $\mathrm{SFLASH}^{v 2}$, which is known for its celerity. The sample scheme TTS $(20,28)$ is fast enough for practical deployment on a low-end 8051-based embedded device. A really low-end part like a stock Intel $8051 \mathrm{AH}$ running at $3.57 \mathrm{MHz}$ can sign in just 170ms. A better 8051-compatible chip will take a lot less time.

Security requirements today demand on-card key generation, and the big public keys of a multivariate PKC create a storage problem. TTS is unusual in that public keys can be synthesized on-card at a decent pace for block-by-block output, using some minimal information kept on-card. Since this does not take much more time than the I/O needed to transmit the public key to a reader, we can avoid holding the entire public key in the limited memory of a smart card. We show that this to be a gain for multivariate PKC's with relatively few terms per central equation. The literature is not rich in this kind of detailed description of an implementation of a signature scheme - capable of fast on-card public key generation, on a low-cost smart card without a co-processor, and at NESSIE-approved security levels.

We look into other theory issues like safeguarding against side-channel attacks, and using unusual techniques for linear algebra under serious space restrictions, which may help implementations of other multivariate PKC's such as SFLASH.
\end{abstract}

Keywords: Multivariate public-key cryptosystem, finite field, smart card, 8051.

\section{Introduction}

For most adopters of Public-Key Infrastructure, the quarter-century-old RSA still remains the public-key cryptosystem of choice. We see that all is not perfect:

RSA is too slow to be used on a smart card and this keeps the security achieved by smart card solutions insufficient: unable to implement a real public key signature. ... N. Courtois et al ([1], 2003).

This must be taken in context as a historical perspective: Acceptable signing speed on smart cards with reasonably long RSA keys has become feasible around the turn of the millennium, especially with special-purpose hardware co-processors. 
However, cost of deployment is still an obstacle, and there is clearly room for improvement: Chips must get even faster and cheaper, or the algorithms need revamping.

Traditionally, Public-Key Infrastructure (PKI) implementers stick to well-established Public-Key Cryptosystems (PKC) based on RSA, occasionally ECC. However these are comparatively slow, so cryptologists sought faster alternatives, among which are the "multivariate PKC", cryptosystems that use substitution into quadratic polynomials as a public map. The currently best known scheme of this type is $\operatorname{SFLASH}^{v 2}$ ([15]), a derivative ([14]) of the prototype $C^{*}$ ([9], broken in 1995 by [12]). Multivariate cryptosystems (more literature on the extended family: 35.78 ) are usually conceded to be faster than RSA and other traditional alternatives with a large algebraic structure. Unfortunately, the only such scheme to get a mention in the recently announced NESSIE final recommendations ([11]) is $\mathrm{SFLASH}^{v 2}$, and only grudgingly:

... not recommended for general use but this signature scheme is very efficient on low cost smart cards, where the size of the public key is not a constraint.

Granted NESSIE was more concerned about SFLASH's untried security than size of its public keys, but still this rather sells SFLASH ${ }^{v 2}$ and other multivariate PKC's short.

We aim to provide another example of how a multivariate $\mathrm{PKC}$ can provide superior performance for less. Our sample scheme, unlike the $C^{*-}$-derived SFLASH ${ }^{v 2}$, is a digital signature scheme from the different family of TTS ([217). Techniques shown here are applicable to other multivariate PKC's, however. Our test results on 8051-compatible microcontrollers are tabulated along with data published by the NESSIE project ([11]) and other recent sources (e.g. [1]). It showcases TTS well:

Table 1. 8051-compatible results for various digital signature schemes $(*=$ with co-processor $)$

\begin{tabular}{|c|c|c|c|c|c|c|}
\hline Scheme & Platform (T number) & Clock & Pr. Key & Code & RAM & Signature \\
\hline \multirow{3}{*}{ TTS $(20,28)$} & Intel 8032AH (12) & \multirow{5}{*}{$3.57 \mathrm{MHz}$} & \multirow{3}{*}{$1.4 \mathrm{kB}$} & $1.4 \mathrm{kB}$ & \multirow{3}{*}{$128 \mathrm{~B}$} & $144 \mathrm{~ms}$ \\
\hline & Intel 8051AH (12) & & & $16 \mathrm{kB}$ & & $170 \mathrm{~ms}$ \\
\hline & Winbond W77E58 (4) & & & $1.6 \mathrm{kB}$ & & $64 \mathrm{~ms}$ \\
\hline ESIGN & \multirow{2}{*}{ Intel 8051AH (12) } & & $336 \mathrm{~B}$ & $3.0 \mathrm{kB}$ & $800 \mathrm{~B}$ & $12.0 \mathrm{~s}$ \\
\hline \multirow{2}{*}{$\mathrm{SFLASH}^{v 2}$} & & & \multirow{2}{*}{$2.4 \mathrm{kB}$} & \multirow{2}{*}{$3.3 \mathrm{kB}$} & \multirow{2}{*}{$344 \mathrm{~B}$} & $1.07 \mathrm{~s}$ \\
\hline & \multirow{2}{*}{ Infineon SLE66 (2) } & \multirow{2}{*}{$10 \mathrm{MHz}$} & & & & $59 \mathrm{~ms}$ \\
\hline & & & \multirow{3}{*}{$320 \mathrm{~B}$} & \multirow{5}{*}{ N/A } & \multirow{5}{*}{$\geq 1 \mathrm{kB}$} & many s \\
\hline $\begin{array}{l}\text { RSA-PSS } \\
(1024 \text { hits) }\end{array}$ & NEC $\mu$ PD789828*(12) & $40 \mathrm{MHz}$ & & & & $100 \mathrm{~ms}$ \\
\hline & \multirow{3}{*}{ Infineon SLE66*(2) } & \multirow{2}{*}{$5 \mathrm{MHz}$} & & & & $230 \mathrm{~ms}$ \\
\hline RSA-2048 & & & $640 \mathrm{~B}$ & & & $1.1 \mathrm{~s}$ \\
\hline ECDSA-191 & & $10 \mathrm{MHz}$ & $24 \mathrm{~B}$ & & & $180 \mathrm{~ms}$ \\
\hline NTRU-Sign & Philips 8051 (6) & $16 \mathrm{MHz}$ & $100 \mathrm{~B}$ & $5 \mathrm{kB}$ & N/A & $160 \mathrm{~ms}$ \\
\hline
\end{tabular}

Special Note: 8051-compatible parts running at the same nominal clock frequency have widely divergent speeds on the same program. On every 8051-compatible part, a majority of common instructions execute in the same amount of time, which is called the instruction cycle for this part. Every instruction executes in an integral number of instruction cycles. The ratio of one instruction cycle to one clock cycle is called the "T number". So a Siemens-Infineon SLE66 is up to six and usually 4-5 times faster than a 8051 at the same clock rate. Some platforms also have extras goodies including cryptographic co-processors, so some care is needed in interpreting the tabulated results. 
Sometimes TTS $(20,28)$ need to hash just once while ESIGN or SFLASH ${ }^{v 2}$, repeated hashes must be taken. Without worrying about details, all told TTS $(20,28)$ is 6 times faster on the same resources than $\mathrm{SFLASH}^{v 2}$, which in turn was noted for being faster without a coprocessor than the traditional alternatives with a coprocessor.

This paper details an implementation of $\operatorname{TTS}(20,28)$ on an 8051 compatible microcontroller. Sec.2] describes the mathematics. Sec.3 summarizes the 8051 platform (with a more complete review provided in Appendix A. Sec. 4 and in particular Secs.4.44.6 give the innards of the algorithm. Sec. 5 discusses technical issues, including sidechannel attack concerns. Some unusual maneuvers in linear algebra help to manage the large amounts of data efficiently during key generation on RAM-poor smart cards. We also explain why TTS can sign and generate keys quickly and efficiently. The real-time on-card public key generation capability ameliorates a problem affecting most multivariates even when the keys are not stored on-card, i.e., the large public key makes on-card key generation frequently infeasible and key management difficult (cf. [16]).

\section{Principles of TTS and Our Sample Scheme TTS $(20,28)$}

In a multivariate PKC, usually the public map is a composition $V=\phi_{3} \circ \phi_{2} \circ \phi_{1}$ with both $\phi_{1}: \mathbf{w} \mapsto \mathbf{x}=\mathrm{M}_{1} \mathbf{w}+\mathbf{c}_{1}$ and $\phi_{3}: \mathbf{y} \mapsto \mathbf{z}=\mathrm{M}_{3} \mathbf{y}+\mathbf{c}_{3}$ being affine and invertible. All arithmetic is done over a finite field (the base field) which in TTS is $K=\operatorname{GF}\left(2^{8}\right)$.

A digital signature scheme is considered to be in the TTS family ([2]) if $\phi_{2}$ is a tame map, a polynomial map with relatively few terms in the equations, easily invertible through serial substitution or solution of linear equations, but without a low degree explicit inverse. Tame maps extend the concept of Tame Transformations from algebraic geometry ([10]), and may be said to combine the traits of triangular constructs (introduced to cryptography in [5], cf. also [617]) and Oil-and-Vinegar ([7,8]).

We refer the reader to [2] for some background, and [17] for a topical assessment of TTS. We will use for illustration the TTS instance exhibited in [17] with the following central map $\phi_{2}: \mathbf{x}=\left(x_{0}, x_{1}, \ldots, x_{27}\right) \mapsto \mathbf{y}=\left(y_{8}, y_{9}, \ldots, y_{27}\right)$ :

$$
\begin{aligned}
y_{i}=x_{i}+ & \sum_{j=1}^{7} p_{i j} x_{j} x_{8+(i+j \bmod 9)}, i=8 \cdots 16 \\
y_{17}=x_{17} & +p_{17,1} x_{1} x_{6}+p_{17,2} x_{2} x_{5}+p_{17,3} x_{3} x_{4} \\
& +p_{17,4} x_{9} x_{16}+p_{17,5} x_{10} x_{15}+p_{17,6} x_{11} x_{14}+p_{17,7} x_{12} x_{13} \\
y_{18}=x_{18} & +p_{18,1} x_{2} x_{7}+p_{18,2} x_{3} x_{6}+p_{18,3} x_{4} x_{5} \\
& +p_{18,4} x_{10} x_{17}+p_{18,5} x_{11} x_{16}+p_{18,6} x_{12} x_{15}+p_{18,7} x_{13} x_{14} \\
y_{i}=x_{i}+ & p_{i, 0} x_{i-11} x_{i-9}+\sum_{j=19}^{i} p_{i, j-18} x_{2(i-j)} x_{j} \\
& +\sum_{j=i+1}^{27} p_{i, j-18} x_{i-j+19} x_{j}, i=19 \cdots 27 .
\end{aligned}
$$

This central map works with 20-byte hashes and 28-byte signatures, and the corresponding TTS instance will be henceforth called TTS $(20,28)$.

To Generate Keys: Assign non-zero random values in $K=\mathrm{GF}\left(2^{8}\right)$ to parameters $p_{i j}$; generate random nonsingular matrices $\mathrm{M}_{1} \in K^{28 \times 28}$ and $\mathrm{M}_{3} \in K^{20 \times 20}$ (usually via LU decomposition) and vector $\mathbf{c}_{1} \in K^{28}$. Compose $V=\phi_{3} \circ \phi_{2} \circ \phi_{1}$; assign $\mathbf{c}_{3} \in K^{20}$ so that $V$ has no constant part. Save quadratic and linear coefficients of $V$ as public key (8680 bytes). Find $\mathrm{M}_{1}^{-1}, \mathrm{M}_{3}^{-1}$; save them with $\mathbf{c}_{1}, \mathbf{c}_{3}$, and the parameters $p_{i j}$ as the private key (1399 bytes). 
To Sign: From the message $M$, first take its digest $\mathbf{z}=H(M) \in K^{20}$, then compute $\mathbf{y}=\mathrm{M}_{3}^{-1}\left(\mathbf{z}-\mathbf{c}_{3}\right)$, then compute a possible $\mathbf{x} \in \phi_{2}^{-1}(\mathbf{y})$ as follows:

1. Randomly assign $x_{1}, \ldots, x_{7}$ and try to solve for $x_{8}$ to $x_{16}$ in the first 9 equations. Since the determinant (for any $x_{2} \cdots x_{7}$ ) of this system is a degree-9 polynomial in $x_{1}$, there can only be at most 9 choices of $x_{1}$ out of 256 to make the first system degenerate. Keep trying until we find a solution.

2. Solve serially for $x_{17}$ and $x_{18}$ using the next two equations ( $y_{17}$ and $y_{18}$ ).

3. Assign a random $x_{0}$ and try to solve for $x_{19}$ through $x_{27}$ from the last 9 equations. Again, at most 9 values of $x_{0}$ can make the determinant of the system zero. So keep trying new values of $x_{0}$ until a solution is found.

Our desired signature is $\mathbf{w}=\mathrm{M}_{1}^{-1}\left(\mathbf{x}-\mathbf{c}_{1}\right)$. Release $(M, \mathbf{w})$.

To Verify: On receiving $(M, \mathbf{w})$, compute $\mathbf{z}=H(M)$ and match with $V(\mathbf{w})$.

\section{Summary of the 8051 Hardware Platform}

The reader may find the details of the key-generation and signing processes (particularly those of Sec.4.5) tedious, but all the contortions are necessitated by the fact that EEPROM memory cannot be reliably read from soon after it is written to. This is not uncommon in the embedded realm. This section provides an executive summary for those unfamiliar with the 8051 chip. Those who are already familiar with the 8051 can skip the rest of this section. Those interested in such things can please refer to the appendix.

Memory: The 8051 has 128 bytes of fast directly-addressable RAM on-board, called data. Some data locations are used as architectural stack and registers including the important registers R0 and R1. The rest hold important system and user data. Most extant 8051-compatibles have 128 bytes more fast RAM onboard, only addressable indirectly through the registers $\mathrm{R} 0$ and R1. As the data can also be accessed this way, all 256 bytes are together called idata. In theory the 8051 can address $64 \mathrm{kB}$ of immutable memory (code) and $64 \mathrm{kB}$ of off-board RAM or EEPROM (xdata), both indirectly using the DPTR register. The code cannot be written to and can be accessed with an offset for table lookups. In practice usually all external memory are accessed identically. Accessing code and xdata takes twice as much time as data and idata besides being harder to set up for.

EEPROM and flash memory are accessed just like RAM except that one must wait, usually for a few milliseconds, before accessing a recently written EEPROM location. A write followed too soon by another access results in an error.

ALU: The Arithmetic-Logic Unit has many specialized registers (the most important being the accumulator A and the Data Pointer DPTR) and instructions. There is an integer multiply and divide, and instruction to increment - but not decrement DPTR, so large arrays are best accessed in increasing order. Each instructions execute serially in a fixed number of instruction cycles (cf. paragraph after Tab. 11.

Resources: I/O is through specialized latches, and communication to a computer or other device must be done through a reader unit, often attached to a PC on a USB port. The access is serial and slow say $1 \mathrm{kB} / \mathrm{s}$. Hardware random-number generation can be added cheaply to most cards. Cheap smart cards today have $4 \mathrm{kB}$ or more in ROM and EEPROM, and a little RAM - sometimes $256 \mathrm{~B}$, frequently $0.5 \mathrm{kB}$, sometimes $1 \mathrm{kB}$. $1.5 \mathrm{kB}$ or more RAM is mostly available in heavyweight cards. 


\section{Actual Performance and Implementation Details}

We recompiled portable C code for $\operatorname{TTS}(20,28)$ (cf. [17]), with C51, a popular embedded compiler. A few routines were rewritten in assembly. Test results from standard development kits are given in Sec. 4.2 and implementation details in Sec.4.4 4.6.

\subsection{Hardware Resource Requirements}

As mentioned in Appendix A.3, a low-end card can either just sign or do both key generation and signing. We list our requirements in RAM (data, idata and external RAM or xdata, see Appendix A.1 , EEPROM, total ROM, etc.:

To Sign: 128 bytes of RAM (19 bytes of data for state, 109 more bytes in data/idata or xdata for temporary storage), $1.3 \mathrm{kB}$ of EEPROM for private keys, $1.6 \mathrm{kB}$ of further code space $(0.8 \mathrm{kB}$ of program, $0.8 \mathrm{kB}$ of tables). For controllers with 256 bytes of on-board RAM (idata), it is an option to keep all the data for the Gaussian elimination in the idata, which means shorter code (no need to move things between idata and xdata) at $1.4 \mathrm{kB}(0.2 \mathrm{kB}$ less $)$ and at least a $12 \%$ speed up. Since we must have some RAM to put the matrix for the elimination stage, a plain-vanilla Intel 8051 will be assumed to have least 128 bytes of xdata RAM storage.

Both To Sign and To Generate Keys: There are two choices:

- On EEPROM-poor cards, we do not store the entire public key. During setup only the private key and some critical intermediate data are generated and stored in EEPROM, enough that chunks of the public key can be computed and output on-the-fly as requested. This requires $2.7 \mathrm{kB}$ of EEPROM (1399B in the private key plus 1212B in intermediate data, plus some left-over wasted space) plus $4.2 \mathrm{kB}$ more code space (in ROM or EEPROM) is required. There is $3.8 \mathrm{kB}$ in the program for both key generation and signing, $0.4 \mathrm{kB}$ in subsidiary $\mathrm{I} / \mathrm{O}$, including $0.8 \mathrm{kB}$ for tables as above.

- We can compute and store the entire public key for later retrieval. This takes $11.3 \mathrm{kB}$ of EEPROM space, plus $4.2 \mathrm{kB}$ more ROM or EEPROM code space.

In both cases we need 128 bytes in data, idata or xdata storage. If we need blockwrites to EEPROM or do block-outputs from the smart card, we will also need 128 more bytes of RAM for buffer. When we can afford to usually we do the entire Gaussian elimination from idata. PC access provided through USB-port device.

\subsection{Performance Data and Brief Description of Programs}

The signing portion of the program is straightforward and can be implemented straight out of Sec. [2] On average, signing takes about 170ms on a $3.57 \mathrm{MHz}$ stock Intel $8051 \mathrm{AH}$ (a really low-end part). Every other part is faster than the 8051AH. The same code running on a $3.57 \mathrm{MHz}$ ("4T") WinBond W77E58 only takes $64 \mathrm{~ms}$.

For reference, of the $170 \mathrm{~ms}$ average time taken by the signing operation on the Intel $8031 / 32$ at $3.57 \mathrm{MHz}$ is divided as follows: The $\phi_{3}$ portion takes $34 \mathrm{~ms}, \phi_{2} 71 \mathrm{~ms}$, and $\phi_{1} 65 \mathrm{~ms}$. On a Winbond W77E58, the times are $13 \mathrm{~ms}, 27 \mathrm{~ms}, 24 \mathrm{~ms}$ respectively. Using $10 \mathrm{MHz}$ parts, the speedup is almost linear. The W77E58 takes about $23 \mathrm{~ms}$ to sign and the Intel $8032 \mathrm{AH}$ takes $61 \mathrm{~ms}-51 \mathrm{~ms}$ if we run the entire elimination from idata. 
Once we get to $16 \mathrm{MHz}$ or faster clocks, some instructions require extra instruction cycles to execute, and I/O times start to dominate, so the scaling is a lot less than linear.

The process for key generation is a lot more complicated and slower than signing. When the smart card is initialized, we must first generate $M_{1}, M_{1}^{-1}, M_{3}, M_{3}^{-1}$ via LU decomposition, store to EEPROM, then generate $\left(p_{i j}\right)_{8 \leq i \leq 27}$ and $\mathbf{c}_{1}$, compute $\mathbf{c}_{3}$ and store everything in EEPROM along the way. Note that $\mathbf{c}_{3}=\mathrm{M}_{3}\left(\phi_{2}\left(\mathbf{c}_{1}\right)\right)$ and hence:

$$
\left(\mathbf{c}_{3}\right)_{k}=\sum_{\ell=n-m}^{n-1}\left[\left(\mathrm{M}_{3}\right)_{k,(\ell-n+m)}\left(\left(\mathbf{c}_{1}\right)_{\ell}+\sum_{p x_{\alpha} x_{\beta} \text { in } y_{\ell}} p\left(\mathbf{c}_{1}\right)_{\alpha}\left(\mathbf{c}_{1}\right)_{\beta}\right)\right] .
$$

The sum is over each term $p x_{\alpha} x_{\beta}$ in the equation for $y_{\ell}$. We may end the setup process here, and the generated information is enough to compute the coefficients of the public key polynomials, 20 at a time. In this mode, the card awaits an appropriate outside input before signing or computing and emitting the public key in small chunks on-the-fly.

Setting up on an Intel $8032 \mathrm{AH}$ at $3.57 \mathrm{MHz}$ (computing the private key and intermediate data) takes 7.7 seconds, including a little error checking. The process takes 3.6 seconds on a $3.57 \mathrm{MHz}$ Winbond W77E58.

The rest of public-key generation is to compute for each $(i, j)$ the coefficients of $w_{i} w_{j}$ or $w_{i}^{2}$ or $w_{i}$ in $z_{k}$ for every $k$ at once. To show that this is possible, we will follow Imai and Matsumoto ([9]) and divide the coefficients involved in each public key polynomial into linear, square, and crossterm portions as follows:

$$
z_{k}=\sum_{i} P_{i k} w_{i}+\sum_{i} Q_{i k} w_{i}^{2}+\sum_{i>j} R_{i j k} w_{i} w_{j}
$$

The coefficients $\left(P_{i k}, Q_{i k}, R_{i j k}\right)$ are related to $\mathrm{M}_{1}, \mathrm{M}_{3}, \mathbf{c}_{1}$, and the parameters $\left(p_{i j}\right)_{8 \leq i \leq 27}$ as follows, where each sum is over the terms $p x_{\alpha} x_{\beta}$ in the equation for $y_{\ell}$ :

$$
\begin{aligned}
P_{i k} & \left.=\sum_{\ell=n-m}^{n-1}\left[\left(\mathrm{M}_{3}\right)_{k,(\ell-n+m)}\left(\mathrm{M}_{1}\right)_{\ell i}+\sum_{p x_{\alpha} x_{\beta} \text { in } y_{\ell}} p\left(\left(\mathrm{M}_{1}\right)_{\alpha i}\left(\mathbf{c}_{1}\right)_{\beta}+\left(\mathbf{c}_{1}\right)_{\alpha}\left(\mathrm{M}_{1}\right)_{\beta i}\right)\right)\right] \\
Q_{i k} & =\sum_{\ell=n-m}^{n-1}\left[\left(\mathrm{M}_{3}\right)_{k,(\ell-n+m)}\left(\sum_{p x_{\alpha} x_{\beta} \text { in } y_{\ell}} p\left(\mathrm{M}_{1}\right)_{\alpha i}\left(\mathrm{M}_{1}\right)_{\beta i}\right)\right] \\
R_{i j k} & =\sum_{\ell=n-m}^{n-1}\left[\left(\mathrm{M}_{3}\right)_{k,(\ell-n+m)}\left(\sum_{p x_{\alpha} x_{\beta} \text { in } y_{\ell}} p\left(\left(\mathrm{M}_{1}\right)_{\alpha i}\left(\mathrm{M}_{1}\right)_{\beta j}+\left(\mathrm{M}_{1}\right)_{\alpha j}\left(\mathrm{M}_{1}\right)_{\beta i}\right)\right)\right]
\end{aligned}
$$

Here $m=20, n=28$. For a smart card equipped with a lot of EEPROM or flash, we need not compute and emit the public key piecemeal. It is possible to compute everything right there and write everything to EEPROM, to be read at a later time.

A $3.57 \mathrm{MHz}$ Intel 8051 or $8032 \mathrm{AH}$ averages about $150 \mathrm{~ms}$ to generate a 20-byte block of the public key from the intermediate data and signal that it is ready to send. On a $3.57 \mathrm{MHz}$ Winbond W77E58 with sufficient storage, generating the entire public key takes 33 seconds. It takes some 15 seconds to transmit everything from card to PC. 


\subsection{Finite Field Arithmetic and Other Details}

As in any other multivariate PKC, we need to represent each element of $\mathrm{GF}\left(2^{8}\right)$ as an integer between 0 and 255 (an unsigned char). We choose the "Standard" representation used by AES ([4]), but we could choose any encoding as long as addition can be represented as a bitwise xor.

A standard way of implementing finite field multiplication is to choose a fixed primitive element $g \in \mathrm{GF}\left(2^{8}\right)$ and store logarithm and exponential look-up tables in base $g$, intending to multiply non-zero $x$ and $y$ as $x y=g^{\left(\log _{g} x+\log _{g} y\right) \bmod 255}$. We will do a lot of manipulations of data in log-form, which means we represent the zero of the field $\mathrm{GF}\left(2^{8}\right)$ by the byte 0 , and any other field element $a$ by the unique positive integer $x \leq 255$ that satisfies $a=g^{x}$. Note: 1 is represented as $g^{255}$, not $g^{0}$.

In implementing the signing portion of the algorithm, we need the following data in ROM: 256-byte log-table in base $g$; 512-byte table of exponentiation $\left(x \mapsto g^{x}\right)$, this can be shortened to 256 bytes at a roughly $15 \%$ speed penalty.

The private key comprises the matrices $\left(\mathrm{M}_{1}^{T}\right)^{-1}$ and $\left(\mathrm{M}_{3}^{T}\right)^{-1}$, parameters $\left(p_{i j}\right)$ of the central map, and the vectors $\mathbf{c}_{1}, \mathbf{c}_{3}$. We store everything except the vectors $\mathbf{c}_{1}$ and $\mathbf{c}_{3}$ in $\log _{g}$-form, and the matrices column-first (as indicated by the transposed notation).

The intermediate key-generation data are $\mathrm{M}_{1}^{T}, \mathrm{M}_{3}^{T}$ (in column-first, $\log _{g}$-form), and a componentwise $\log$ of $\mathbf{c}_{1}$. The public key consists of coefficients $\left(P_{i k}\right),\left(Q_{i k}\right),\left(R_{i j k}\right)_{i>j}$, with each block arranged in order of increasing $i, j$, then $k$.

\subsection{The Signing Process}

The actual signing program operates on a 20-byte array $\mathbf{z}$ in idata in three stages, corresponding to $\phi_{1}^{-1}, \phi_{2}^{-1}$, and $\phi_{3}^{-1}$. Due to the amount of array access in the Gaussian elimination, $\phi_{2}^{-1}$ takes most of the time. If we put the entire system matrix in idata we can save at least 10 percent upwards of running time, but most often we forego that and do it mostly from xdata due to memory resource problems.

1. Do $\left(\phi_{3}\right)^{-1}$, which is essentially a matrix multiplication, as follows:

a) Zero out a 20-byte area $\mathbf{y}$ and replace $\mathbf{z}$ by $\mathbf{z}^{\prime}$, the componentwise $\log$ of $\mathbf{c}_{3}+\mathbf{z}$;

b) looping over $i=19,18 \cdots 0$ and do the following loop if $z_{i}^{\prime} \neq 0$ :

c) Looping over $j=19 \cdots 0$, when $\left(\mathrm{M}_{3}\right)_{j i} \neq 0$, add (xor) $g^{\left(z_{i}^{\prime}+\log _{g}\left(\mathrm{M}_{3}\right)_{j i}\right) \bmod 255}$ into $y_{j+8}$. Note: $\mathrm{M}_{3}$ is stored in log-form and transposed so that it can be accessed sequentially, and we can compute $(R+\mathrm{A})$ mod 255 in only two instructions: add A, $R$ (add register $R$ to the accumulator A) then adc A, \#0 (add the carry from the last add into accumulator).

The inner loop of this routine reads coefficients off a table, multiplies to a variable, then adds them to different components of a vector (cf. also Sec. 5.3).

2. Do $\left(\phi_{2}\right)^{-1}$, which is performed as follows:

a) For $i=1 \cdots 7$, generate randomly and save (in an idata array) $\log _{g} x_{i}$, again with the proviso that 0 represents 0 , not 1 which is represented by 255 .

b) Establish in a 90-byte block BA (in xdata or idata) of RAM the first linear system to be solved for $x_{8}, \ldots, x_{16}$ by doing the following for $i=8 \cdots 16$ :

i. The constant of each equation location $(\mathrm{BA}[10(i-8)+9])$ is filled with $y_{i}$.

\footnotetext{
${ }^{1}$ We chose as $g$ the canonical generator of the AES field representation.
} 
ii. Looping over $j=1 . .7$, insert into the location corresponding to the coefficient of $x_{(i+j(\bmod 9))+8}($ location BA $[10(i-8)+(i+j(\bmod 9))])$.

iii. xor $\mathrm{BA}[10(i-8)+(i+1(\bmod 9))]$ with 1 .

iv. Let $\mathrm{BA}[10(i-8)+(i+8(\bmod 9))]=\mathrm{BA}[10(i-8)+(i+9(\bmod 9))]=0$.

c) Run elimination on 9 variables to get $x_{8}, \ldots, x_{16}$, then find $x_{17}$ and $x_{18}$ by solving for them in the next two equations (all $x_{i}$ will be stored in log-form).

d) Establish another system of equations in BA by looping over $i=19 \cdots 27$ :

i. Insert $y_{i}+p_{i 0} x_{i-11} x_{i-9}$ as the constant term (location BA $[10(i-19)+9]$ ).

ii. Looping over $j=0 \cdots i-19$, let BA $[10(i-19)+j]=p_{i, j+1} x_{2(i-j-19)}$.

iii. Looping over $j=i-19 \cdots 8$, let $\mathrm{BA}[10(i-19)+j]=p_{i, j+1} x_{i-j}$.

iv. xor $\mathrm{BA}[10(i-8)+(i+1(\bmod 9))]$ with 1 .

e) Run elimination on 9 variables to obtain $x_{19}, \ldots, x_{27}$ (again in log-form).

3. Do $\left(\phi_{1}\right)^{-1}$, another matrix multiplication like $\left(\phi_{3}\right)^{-1}$, with different parameters.

\subsection{Key Generation, First Half: Generating $M_{1}, M_{3}$, and Their Inverses}

The following routine computes and stores $\mathrm{M}_{1}^{T},\left(\mathrm{M}_{1}^{T}\right)^{-1}, \mathrm{M}_{3}^{T},\left(\mathrm{M}_{3}^{T}\right)^{-1},\left(p_{i j}\right)$ for $8 \leq$ $i \leq 18,1 \leq j \leq 6$ and $19 \leq i \leq 27,0 \leq j \leq 9, \mathbf{c}_{1}, \log _{g} \mathbf{c}_{1}$, and $\mathbf{c}_{3}$. Total EEPROM space required is 2768 bytes, with 1399 bytes in private keys $\left(\left(M_{1}^{T}\right)^{-1},\left(M_{3}^{T}\right)^{-1}\right.$, the $\left.\left(p_{i j}\right), \mathbf{c}_{1}, \mathbf{c}_{3}\right)$ and 1212 bytes of intermediate data to be used to produce the public keys. There are 157 bytes used and erased. No more RAM than the 256 bytes of idata is needed; in fact, only 128 bytes are necessary if a write buffer is not needed. Of course, extra RAM helps. Recall that matrices $M_{1}, M_{1}^{-1}, M_{3}, M_{3}^{-1}$ are stored transposed and in log-form (cf. Sec.4.3) for convenience.

1. Generate matrices $M_{1}$ and $\left(M_{1}\right)^{-1}$ via LU decomposition.

a) Generate and write to EEPROM entries (in log-form) of the diagonal matrix $D_{1}$ (28 non-zero bytes), the lower triangular matrix $L_{1}(28 \times 27 / 2=378$ bytes, also in log-form), and the upper triangular matrix $U_{1}$ (same as above), and do so in the area from the 1569 th to 2352 th bytes from the beginning (hence, leaving the first 1568 bytes empty). The entries of $L$ and $U$ are generated in an unusual format. We line $L$ up column-first, but $U$ will be in column-first but reverse order, i.e.: $L_{10}, L_{20}, \ldots, L_{n-1,0}, L_{21}, \ldots, L_{n-1,1}, \ldots, L_{n-1, n-2}$ and $U_{n-2, n-1}, \ldots, U_{0, n-1}, U_{n-3, n-2}, \ldots, U_{0, n-2}, \ldots, U_{23}, U_{13}, U_{12}$.

b) Invert $D_{1}, L_{1}, U_{1}$ and write to EEPROM (in the first 784 bytes). Inverting $D_{1}$ is easy. We invert $L_{1}$ into $L_{1}^{-1}$ (stored in the same format) as follows:

i. Repeat (ii.-v.) over $i=1,2, \ldots, n-1$ :

ii. Read $\left[\left(L_{1}\right)_{i, i-1}, \ldots,\left(L_{1}\right)_{n-1, i-1}\right]$ from xdata into $\left[z_{i}, \ldots, z_{n}\right]$ in idata.

iii. For each $j=i, \ldots, n-1$ where $z_{j} \neq 0$, replace $z_{j}$ by $\left(\log _{g} z_{j}\right)$ and do:

iv. For each $k=j+1, \ldots, n-1$ such that $L_{k j} \neq 0$, add $g^{m+L_{k j}}$ to $z_{k}$.

v. Now $\left[z_{i}, \ldots, z_{n}\right]$ is the column $\left[\log _{g}\left(L_{1}^{-1}\right)_{i, i-1}, \ldots, \log _{g}\left(L_{1}^{-1}\right)_{n-1, i-1}\right]$. Write to EEPROM, or (for parts with special EEPROM/flash writing rules) copy to a 128-byte buffer and block-write only if the buffer is full.

The same subroutine can invert $U_{1}$ into $U_{1}^{-1}$ in the same inverted column order.

c) Compute $\mathrm{M}_{1}^{-1}=U_{1}^{-1} D_{1}^{-1} L_{1}^{-1}$ and write to EEPROM in the next 784 bytes:

i. $\operatorname{Read}\left[\log _{g}\left(\left(D_{1}^{-1}\right)_{j j}\right)\right]$ into array $\left[d_{j}\right]$ in idata; repeat (ii.-v.) for $i<n$. 
ii. Zero out $z_{0}$ to $z_{i-1}$ (array $z_{0}, \ldots, z_{n-1}$ is in idata); let $z_{i}=d_{j}$; for $i+1 \leq j \leq n-1$ let $z_{j}=\left(d_{j}+\log _{g}\left(L_{1}^{-1}\right)_{j i}\right) \bmod 255$. Note (cf. 1b) that $\log \left(L_{1}^{-1}\right)_{j i}$ was stored serially.

iii. Looping over $j=i, i+1, \ldots, n-1$, do the following:

iv. For $k=0, \ldots, j-1$, add $g^{\left(z_{j}+\log _{g}\left(U_{1}^{-1}\right)_{k j}\right) \bmod 255}$ into $z_{k}$. Note that $U_{1}^{-1}$ is in reverse order. After the $k$-loop, replace $z_{j}$ by $g^{z_{j}}$.

v. Now (end of $j$-loop) the $\left[z_{j}\right]$ array holds the $i$-th column of $\mathrm{M}_{1}$. Take the componentwise log, then write appropriately into EEPROM (cf.1b).

Note that we used $n$ instead of 28 because the same routines are used for $\mathrm{M}_{3}$.

d) Erase the first 784 bytes, the memory block used for $D_{1}^{-1}, L_{1}^{-1}, U_{1}^{-1}$.

e) Compute (and write out to the freshly erased block of 784 bytes) $\mathrm{M}_{1}=L_{1} D_{1} U_{1}$.

i. Read $\left[\log _{g}\left(\left(D_{1}\right)_{j j}\right)\right]$ into array $\left[d_{j}\right]$ in idata; repeat (ii.-v.) for $i<n$.

ii. Read $\left(\log _{g}\left(U_{1}\right)_{j i}+d_{j}\right) \bmod 255$ to $z_{j}$ (in idata) for $j=0 \cdots i-1$.

iii. Let $z_{i}=d_{i}$. For $j=0 \cdots i$, let $y_{i}=g^{z_{i}}$, and zero out $y_{i+1}, \ldots, y_{n-1}$.

iv. Looping over $0 \leq j \leq i, j \leq k<n$, add $g^{\left(z_{k}+\log _{g}\left(L_{1}\right)_{k j}\right) \bmod 255}$ into $y_{k}$.

v. The $\left(\log y_{k}\right)$ is the $i$-th column of $\log _{g} \mathrm{M}_{1}$, write to EEPROM.

f) Erase the 784-byte EEPROM block used for $D_{1}, L_{1}, U_{1}$.

We should conclude with 1568 bytes of data and 784 freshly erased bytes.

2. Generate $M_{3}$ and $\left(M_{3}\right)^{-1}$ as above, reusing the memory from Step 1 . We should have written 800 bytes of data and have 400 recently erased bytes.

3. Generate and store $\left(p_{i j}\right)$ for $8 \leq i \leq 18,1 \leq j \leq 7$ and $19 \leq i \leq 27,0 \leq j \leq 9$ (167 bytes), reusing the memory from Step 2

4. Generate $\mathbf{c}_{1}$ (28 bytes) and store in space left from Step 2 Compute $\mathbf{c}_{3}$ (20 bytes):

a) Storing (in idata) in the arrays $\mathbf{c}$ and $\mathbf{y}$ the componentwise $\log$ of $\mathbf{c}_{1}$.

b) Reading from the parameter table, compute $\phi_{2}\left(\mathbf{c}_{1}\right)$ by looping over all indices $i=8 \cdots 27$, adding each cross-term into the appropriate $y_{i}$.

c) Write the $\log \left(\mathbf{c}_{1}\right)_{i}$ to EEPROM. Take the logs of $y_{i}$; jump to the multiplication routine in Sec.4.2. The result $\left(\mathbf{c}_{3}\right)$ is written to EEPROM. We are done.

\subsection{Key Generation, Second Half: Computing and Outputting the Public Key}

After the process of Sec. 4.5. we can generate the public key in units of 20 bytes (see Sec.4.2). Generating the public key takes a long time, but the routine itself is simpler:

Generating $R_{i j k}$ : It is more convenient to compute first the coefficients of $w_{i} w_{j}$ in $y_{k}$ :

1. Read in the $i$-th and $j$-th columns of $\log _{g} \mathrm{M}_{1}$ and place in the arrays $\mathbf{c}$ and $\mathbf{c}^{\prime}$.

2. Zero out the array $\mathbf{z}$ and loop over each cross-term $p x_{\alpha} x_{\beta}$ in the equation for $y_{i}$ (reading off parameter table) thusly:

3. Compute $p\left[\left(\mathrm{M}_{1}\right)_{\alpha i}\left(\mathrm{M}_{1}\right)_{\beta j}+\left(\mathrm{M}_{1}\right)_{\alpha j}\left(\mathrm{M}_{1}\right)_{\beta i}\right]$ via $\log _{g}\left(g^{c_{\alpha}+c_{\beta}^{\prime}}+g^{c_{\alpha}^{\prime}+c_{\beta}}\right)$, adding $\log _{g} p$ (read off the parameter table) and exponentiating. Add to $z_{i}$.

4. We now have the coefficient of $x_{i} x_{j}$ in $y_{k}$. Take the logs and multiply by $\mathrm{M}_{3}$ and jump to the matrix multiplication routine (Sec.4.2) to get $R_{i j k}$ for each $k$.

Generating $P_{i k}$ : As above, except for initializing the array $\mathbf{z}$ to $\left[\left(\mathrm{M}_{1}\right)_{k i}\right]_{k=8 \cdots 27}$ instead of zeroing out $\mathbf{z}$, and reading in $\mathbf{c}_{1}$ and the $i$-th column of $\mathrm{M}_{1}$ instead of the $i$-th and $j$-th columns of $\mathrm{M}_{1}$.

Generating $Q_{i k}$ : Like the above, but we only need to read a single column of the $\mathrm{M}_{1}$, and it is faster because there is one fewer add and one fewer multiply in Eq. 4.

After each block of 20 is produced, write it to EEPROM or accumulate for buffered output as needed. Our test code outputs every 6 blocks to get max throughput. 


\section{Discussions and Conclusion}

We discuss some issues germane to our study including side channel attack considerations, optimization, and possible changes to the scheme to suit smart cards better.

\subsection{Why Does TTS Have Faster Key Generation?}

The state-of-the-art in key generation for multivariate PKC's is probably the kind of procedures as given by C. Wolf ([16]). At least, we can find no better, and he managed to save $30 \%$ of running time from previous algorithms. However Wolf states, and it seems commonly agreed to, that computations of the public polynomial using interpolation for large field multivariates, i.e. HFE ([13]) or $C^{*}$-derivatives where the private map really operates on some googol-sized field, take time proportional to $n^{6}$. A cursory look at Eqs. 3 5 will reveal that the number of multiplications in key generation is about $n^{4}$ (really $m^{2} n^{2}$ ) times the average number of terms in an equation in $\phi_{2}$, hence $O\left(n^{4}\right)$. So we expect key generation in TTS to run at about a few hundred times the speed that SFLASH might need if they use the same dimensions.

Timings given in [2,17] support this hypothesis. We do not claim to be anywhere close to as good 8051 programmers as the authors of [1], but the factor of $n^{4}$ vs. $n^{6}$ gives an edge that makes on-card key generation passably quick as opposed to snail-like. In general, a multivariate PKC can be called tame-like ([17]) if its central map has relatively few terms per equation and has a fast inverse. Sec.4.6 and Eqs. 3] 5]demonstrate tamelike-ness are useful for a smart card.

\subsection{Side Channel Attack Considerations}

In [1], the authors discuss defending against a possible differential-power attacks. The structure of SFLASH ${ }^{v}$ is somewhat simpler, but we can take similar precautions against DPA probes to those in [1]. The steps for a DPA-safe signing are:

1. Start out with hash value $\mathbf{z}$. Take a random vector $\mathbf{z}^{\prime} \in K^{m}$. Compute $\mathbf{z}^{\prime \prime}=\mathbf{z}+\mathbf{z}^{\prime}$.

2. Compute $\mathbf{y}^{\prime}=\left(\mathrm{M}_{3}\right)^{-1}\left(\mathbf{z}^{\prime}-\mathbf{c}_{3}\right)$ and $\mathbf{y}^{\prime \prime}=\left(\mathrm{M}_{3}\right)^{-1} \mathbf{z}^{\prime \prime}$. We see that $\mathbf{y}=\mathbf{y}^{\prime}+\mathbf{y}^{\prime \prime}$.

3. Take random bytes $x_{i}^{\prime}, x_{i}^{\prime \prime}$ for $i=0 \cdots 7$. Loop until the systems are solvable.

4. Construct linear systems as in Sec. 4.4, except that we use twice as large a RAM buffer, put in two systems: One filled in using the $x_{i}^{\prime}$ and $y_{i}^{\prime}$, one with the $x_{i}^{\prime \prime}$ and $y_{i}^{\prime \prime}$. Note: Step 2(b)iii of Sec. 4.4 only needs to be performed on one of the matrices.

5. Run a "conjoined Gaussian" with the two matrices. A key operation is division by the sum of the two coefficients at the pivot position. If they are $c_{i i}^{\prime} \neq c_{i i}^{\prime \prime}$, then we can achieve division by the pivot coefficient $\left(c_{i i}^{\prime}+c_{i i}^{\prime \prime}\right)$ through dividing successively with $c_{i i}^{\prime}$ and then by $\left(1+c_{i i}^{\prime \prime} / c_{i i}^{\prime}\right)$. For $9 \times 9$ matrices, this means slightly less than triple the number of multiplications and is time-consuming, but we eventually come down to $\left(x_{8}^{\prime} \cdots x_{16}^{\prime}, x_{8}^{\prime \prime} \cdots x_{16}^{\prime \prime}\right)$, where $x_{i}^{\prime}+x_{i}^{\prime \prime}=x_{i}$.

6. For $i=17$ and 18 , do the following so that $x_{i}=x_{i}^{\prime}+x_{i}^{\prime \prime}$.

$$
x_{i}^{\prime}=y_{i}^{\prime}+\sum_{p x_{\alpha} x_{\beta} \text { in } y_{i}} p \cdot\left(x_{\alpha}^{\prime} x_{\beta}^{\prime}+x_{\alpha}^{\prime \prime} x_{\beta}^{\prime \prime}\right) ; x_{i}^{\prime \prime}=y_{i}^{\prime \prime}+\sum_{p x_{\alpha} x_{\beta} \text { in } y_{i}} p \cdot\left(x_{\alpha}^{\prime \prime} x_{\beta}^{\prime}+x_{\alpha}^{\prime} x_{\beta}^{\prime \prime}\right) .
$$


7. Similarly for $i=19 \cdots 27$ we do the "conjoined Gaussian". We have found $\mathbf{x}^{\prime}$ and $\mathrm{x}^{\prime \prime}$ that sum to the $\mathbf{x}$ of Sec. 2 at about one-quarter speed.

8. Compute $\mathbf{w}^{\prime}=\left(\mathrm{M}_{1}\right)^{-1} \mathbf{x}^{\prime}$ and $\mathbf{w}^{\prime \prime}=\left(\mathrm{M}_{1}\right)^{-1}\left(\mathrm{x}^{\prime \prime}-\mathbf{c}_{1}\right)$. Output $\mathbf{w}=\mathbf{w}^{\prime}+\mathbf{w}^{\prime \prime}$.

Since TTS $(20,28)$, like SFLASH ${ }^{2}$ uses each byte of the entire key continuously, it should be as safe as SFLASH ${ }^{2}$ under the same attacks. The signing code expands to about $3.2 \mathrm{kB}$, and the speed is between a third and a quarter of what it was (still ok). This is obviously not optimal programming and we should be able to optimize it better.

\subsection{Optimization Concerns}

Since we are only doing proof-of-concept work we used C51-compiled code with only a few key routines hand-coded 8051 assembly. This is not the optimal performance available from our hardware, but is a lot better than using just C51, and saves many man-hours compared to doing assembly from the ground up. We feel that we are not giving up much in the way of performance because this is common practice in software design. A local expert on the 8051 offered his opinion that we might be able to improve it by a factor of two in pure assembly. However, the quaint, quirky 8051 architecture severely restricts our options. There is only so much possible with our limited resources, especially the single pointer into xdata. Some notes on optimization possibilities:

- Of particularly note is the idiosyncratic ways the arrays were arranged in memory. We do not have hard proofs but believe that this arrangement is already correctly optimized under the circumstances.

- If the $\mu C$ has access to $1.5 \mathrm{kB}$ of xdata RAM, all the temporary data can stay in RAM and we need not erase from EEPROM at all during key generation. Even 1 kB RAM would make life easier.

- The movx A, @Ri command that use the output latch PO for the high byte of the address line and the register R0 or R1 for the low byte is seldom used, because I/O lines from the outside often get in the way, changing the value of the latch when it shouldn't. With custom solutions, it is possible to have programs up to $20 \%$ faster by using these specialized commands.

- An $n \mapsto \log _{g}\left(1+g^{n}\right)$ table can help. Let this table be E. When computing $R_{i j k}$ (Sec. 4.6), we need to do $\left[\log _{g}\left(g^{c_{\alpha}+c_{\beta}^{\prime}}+g^{c_{\alpha}^{\prime}+c_{\beta}}\right)+\log _{g} p\right] \bmod 255$. Instead, we can save lookups via $\left[\left(c_{\alpha}+c_{\beta}^{\prime}\right)+\mathrm{E}\left[c_{\alpha}^{\prime}+c_{\beta}-\left(c_{\alpha}+c_{\beta}^{\prime}\right)\right]+\log _{g} p\right] \bmod 255$.

- We never really rewrote our programs for parts with dual DPTR's. With proper utilization, this is said to increase the speed by some $25 \%$ for array-heavy code.

- With the base field $K=\mathrm{GF}\left(2^{7}\right)$, the signing can run with the exponential table in idata (did the designer of SFLASH think of this?). More extremely, with $K=$ $\mathrm{GF}\left(2^{6}\right)$, both the log and the exponential table can fit into the idata. We can change to a $\left(\mathrm{GF}\left(2^{7}\right)\right)^{28} \mapsto\left(\mathrm{GF}\left(2^{7}\right)\right)^{36}$ version of TTS for speed if key size and extra RAM storage is not a problem. Preliminary tests show that it is about $13 \%$ faster.

\subsection{Conclusion}

We believe that our test result shows the TTS family of multivariate signature schemes in general and $\operatorname{TTS}(20,28)$ in particular to be very speedy. Not only is it fast, but it also 
consumes little enough resources that it definitely merits further attention as an on-card solution. The family of schemes has great potential for use in low-cost smart cards, especially those without a cryptographic coprocessor.

We note that $\operatorname{TTS}(20,28)$ by current estimates should be at least as secure as RSA1024, and TTS $(24,32)$ at least as secure to RSA-1536 (cf. [17]). Even if this estimate is slightly off, the speed of the implementations should still make TTS quite useful for smart cards. Furthermore, TTS is clearly an extensible system and we did an implementation for the following central map ([17])

$$
\begin{aligned}
y_{i}=x_{i}+ & \sum_{j=1}^{7} p_{i j} x_{j} x_{8+(i+j+1 \bmod 10)}, i=8 \cdots 17 \\
y_{i}=x_{i}+ & p_{i 1} x_{i-17} x_{i-14}+p_{i 2} x_{i-16} x_{i-15}+p_{i 3} x_{i-10} x_{i-1}+p_{i 4} x_{i-9} x_{i-2} \\
& +p_{i 5} x_{i-8} x_{i-3}+p_{i 6} x_{i-7} x_{i-4}+p_{i 7} x_{i-6} x_{i-5}, i=18 \cdots 21 ; \\
y_{i}=x_{i}+ & p_{i, 0} x_{i-10} x_{i-14}+\sum_{j=22}^{i} p_{i, j-21} x_{2(i-j)} x_{j} \\
& +\sum_{j=i+1}^{31} p_{i, j-21} x_{i-j+21} x_{j}, i=22 \cdots 31 .
\end{aligned}
$$

This scheme has $m=24$ (192-bit hashes) and $n=32$. We tabulate our new test results

\begin{tabular}{|c|c|c|c|c|c|c|c|c|}
\hline Scheme & Controller & $\begin{array}{l}\text { PrivKey } \\
\text { Length }\end{array}$ & \begin{tabular}{|c} 
Signing \\
Time
\end{tabular} & $\begin{array}{l}\text { Signing } \\
\text { Code }\end{array}$ & $\begin{array}{l}\text { Keygen } \\
\text { Time }\end{array}$ & $\begin{array}{c}\text { Keygen } \\
\text { Code }\end{array}$ & $\begin{array}{c}\text { Extra } \\
\text { EEPROM }\end{array}$ & $\begin{array}{l}\text { Setup } \\
\text { Time }\end{array}$ \\
\hline \multirow{3}{*}{$\operatorname{TTS}(20,28)$} & $i 8032 \mathrm{AH}$ & \multirow{3}{*}{$1399 \mathrm{~B}$} & $144 \mathrm{~ms}$ & $1.5 \mathrm{kB}$ & \multirow{2}{*}{$78.5 \mathrm{~s}$} & \multirow{6}{*}{$4.2 \mathrm{kB}$} & \multirow{3}{*}{$1.2 \mathrm{kB}$} & \\
\hline & $i 8051 \mathrm{AH}$ & & $170 \mathrm{~ms}$ & & & & & \\
\hline & W77E58 & & $60 \mathrm{~ms}$ & $1.6 \mathrm{kB}$ & $38.3 \mathrm{~s}$ & & & $3.6 \mathrm{~s}$ \\
\hline \multirow{3}{*}{$\operatorname{TTS}(24,32)$} & $i 8032 \mathrm{AH}$ & \multirow{3}{*}{1534 B } & $191 \mathrm{~ms}$ & $1.5 \mathrm{kB}$ & \multirow{3}{*}{$134 \mathrm{~s}$} & & \multirow{3}{*}{$1.6 \mathrm{kB}$} & \\
\hline & $i 8051 \mathrm{AH}$ & & $227 \mathrm{~ms}$ & & & & & \\
\hline & W77E58 & & $85 \mathrm{~ms}$ & $1.0 \mathrm{~KB}$ & & & & $5.4 \mathrm{~s}$ \\
\hline
\end{tabular}
with the old. Again, only the $i 8032 \mathrm{AH}$ code ran the elimination entirely in idata.

As we observed in Sec. 5.3, we expect to improve on our work still. All in all, we think that we have shown TTS and similar variants are worth more attention.

Table 2. Summary for TTS on a 8051

Acknowledgments. We thank Messrs. Po-Yi Huang and Sam Tsai of Solutioninside Inc. for technical assistance, and to Messrs. Bo-Yuan Peng and Hsu-Cheng Tsai of National Taiwan University for commentary and discussion. We thank the anonymous referees for their suggestions and constructive criticism. The first author would also like to thank his beloved Ping.

\section{References}

1. M. Akkar, N. Courtois, R. Duteuil, and L. Goubin, A Fast and Secure Implementation of SFLASH, PKC 2003, LNCS v. 2567, pp. 267-278.

2. J.-M. Chen and B.-Y. Yang, A More Secure and Efficacious TTS Scheme, ICISC '03, LNCS V. 2971, pp. 320-338; full version at http://eprint.iacr.org/2003/160.

3. D. Coppersmith, J. Stern, and S. Vaudenay, The Security of the Birational Permutation Signature Schemes, Journal of Cryptology, 10(3), 1997, pp. 207-221. 
4. J. Daemen and V. Rijmen, The Design of Rijndael, AES - The Advanced Encryption Standard. Springer-Verlag, 2002.

5. H. Fell and W. Diffie, Analysis of a Public Key Approach Based on Polynomial Substitution, CRYPTO'85, LNCS v. 218, pp. 340-349.

6. L. Goubin and N. Courtois, Cryptanalysis of the TTM Cryptosystem, AsIACRYPT 2000, LNCS v. 1976, pp. 44-57.

7. A. Kipnis, J. Patarin, and L. Goubin, Unbalanced Oil and Vinegar Signature Schemes, CRYPTO'99, LNCS v. 1592, pp. 206-222.

8. A. Kipnis and A. Shamir, Cryptanalysis of the Oil and Vinegar Signature Scheme, CRYPTO'98, LNCS v. 1462, pp. 257-266.

9. T. Matsumoto and H. Imai, Public Quadratic Polynomial-Tuples for Efficient SignatureVerification and Message-Encryption, EuROCRYPT'88, LNCS v. 330, pp. 419-453.

10. T. Moh, A Public Key System with Signature and Master Key Functions, Communications in Algebra, 27 (1999), pp. 2207-2222.

11. The NESSIE project webpage: http://www . cryptonessie.org.

12. J. Patarin, Cryptanalysis of the Matsumoto and Imai Public Key Scheme of Eurocrypt'88, CRYPTO'95, LNCS v. 963, pp. 248-261.

13. J. Patarin, Hidden Fields Equations (HFE) and Isomorphisms of Polynomials (IP): Two New Families of Asymmetric Algorithms, EUROCRYPT'96, LNCS v. 1070, pp. 33-48.

14. J. Patarin, L. Goubin, and N. Courtois, $C_{-+}^{*}$ and HM: Variations Around Two Schemes of T. Matsumoto and H. Imai, AsiACRYPT'98, LNCS v. 1514, pp. 35-49.

15. J. Patarin, N. Courtois, and L. Goubin, FLASH, a Fast Multivariate Signature Algorithm, CT-RSA 2001, LNCS v. 2020, pp. 298-307. Updated version available at [11].

16. C. Wolf, Efficient Public Key Generation for Multivariate Cryptosystems, preprint, available at http://eprint.iacr.org/2003/089/.

17. B.-Y. Yang and J.-M. Chen, Rank Attacks and Defence in Tame-Like Multivariate PKC's, see http://eprint.iacr.org/2004/061.

\section{A Architecture of 8051-Based Smart Cards}

We summarize the specifics of our hardware platforms for implementation and testing. Some discussion about clock vs. actual execution speed can be found in Sec.11(following Tab. 372.

\section{A.1 Storage Areas on an 8051-Based Smart Card}

The general structure of an 8051-based 8-bit microcontroller $(\mu C)$ core is the same across implementations. The chip usually has a CPU portion plus some extra memory. On a 8051-like device, we have the following different locations for data storage:

data. The 128 bytes of on-chip high-speed RAM directly addressable by the 8051 core in one instruction cycle. Sometimes referred to as "Register RAM" because in effect they are registers, like the zeroth page of the 6502 and other 8-bit CPUs. A peculiarity of the 8051 instruction set is that some data can be accessed bitwise as flags. This saves valuable data space as well as instructions.

idata. On-chip high-speed RAM that may be accessed in indirect address mode through a byte-long pointer (either of the special registers RO or R1) in one instruction cycle. The data can also be accessed this way and is considered part of the idata. Almost every 8051-compatibles has the 128 extra bytes of idata for 256 bytes total. 
code. ROM, not writable. May be only read indirectly through the Data Pointer (DPTR) register with a fixed latency (normally 2 instruction cycles on a cheap part) via a special movc command. An 8051-like $\mu C$ can address up to $64 \mathrm{kB}$ of code ROM.

xdata. Off-chip read-write memory, accessible only via the movx command, with indirection through either DPTR or a combination of the I/O port register PO and either of the special register R0 and R1. Normally movx takes 2 instruction cycles, but on a high-end part there may be banks of memory with different latency, and a special register to control the access time.

One expect xdata to be RAM, and because the movx/movc commands both set signals lines, effectively adding an extra address line, an 8051-like part can theoretically address $64 \mathrm{kB}$ of ROM (code) and $64 \mathrm{kB}$ of RAM (xdata) for $128 \mathrm{kB}$ memory. However, when the $\mu C$ does not use more than $64 \mathrm{kB}$ total, as a rule the control lines are wired together for convenience 2 , and code and data are read identically. Another important point is that read-write memory can be EEPROM or flash memory as well as RAM:

- RAM for a $\mu C$ is usually costly SRAM. In theory there may be as much as $64 \mathrm{kB}$, but there is often only $256 \mathrm{~B}$, seldom more than $1 \mathrm{kB}$ and almost never more than $2 \mathrm{kB}$. A smart card intended for RSA or ECC work will always have at least $1 \mathrm{kB}$ (usually $1.5 \mathrm{kB})$, because there is a lot of data and co-processors also need memory.

- We will use EEPROM and flash memory as synonyms like most people, although EEPROM can often be written to much faster and far more times than flash.

A $\mu C$ may have no EEPROM at all or as much as $64 \mathrm{kB}$. Reading EEPROM is just like reading off-chip RAM, but completing a write into one EEPROM location takes about $50 \mu \mathrm{s}$ and erasing (resetting it into a writable state) takes much longer, about $5 \mathrm{~ms}$ per access. Often erasure and sometimes writes must be by lines, which are units of access that may be 8 to 128 bytes each. Control signals from the EEPROM can be polled via I/O port latches to tell the programmer whether the EEPROM is ready for writing or successfully written. After an EEPROM address is written to but before the requisite amount of time (some 100 instruction cycles or more) has elapsed, reading that location generates unpredictable results.

There are several modes of writing into EEPROM/flash, and a given part normally does only one. There are 8051 parts with safeguards against loss of power and a block-write operation with a same latency of $5 \mathrm{~ms}$ per 128-byte block, but these tend to be very expensive parts. In parts without the power-failure guard and block-writing features, EEPROM is essentially accessed like RAM, but the program needs to check manually the signal lines. If you are safety-minded, you check first, write, then keep checking the signals to make sure that it is done properly. A more cavalier designer would go about his business after the write is issued, but would presumably do some error-checking.

\section{A.2 The 8051-Based Parts We Tested and Their Characteristics}

Intel 8051AH. One of the original MCS-51 NMOS single-chip 8-bit microcontroller parts, with 32 I/O lines, 2 Timers/Counters, 5 Interrupts/2 Priority Levels, and 128 bytes on-chip RAM (i.e. no extra idata). It has $2 \mathrm{kB}$ of on-chip ROM.

\footnotetext{
${ }^{2}$ movx also can often be faster than movc on high-end parts for a speed advantage.
} 
Intel 8032AH. A stripped-down 8052; just like the $i 8051$ except for having 3 Timers (Counters), 6 Interrupts/4 priority levels, no ROM and 128B more RAM on-chip.

Winbond W77E58. A more upscale CMOS part, with $36 \mathrm{I} / \mathrm{O}$ lines, 3 Timers/Counters, 12 Interrupts/2 priority levels, and 256 bytes on-chip RAM. 32kB ROM built-in. Its big pluses are: Dual Data Pointers (so that using two off-chip memory blocks is a lot faster), $1 \mathrm{kB}$ extra on-chip SRAM (appropriately latched for optimal movx access), and the fact that it is " $4 \mathrm{~T}$ ", i.e., many frequently used instructions takes 4 clock cycles to execute, so it is up to 3 times faster at the same nominal clock.

Dual Data Pointers is a common goodie in high-end 8051-like parts. By toggling a special flag bit, the programmer can switch between two possible DPTR's, which means that the $\mu C$ can quickly access two different look-up tables, among other things.

\section{A.3 Random Number Generation and Other I/O Concerns on an $\mathbf{8 0 5 1}$}

For modern-day PKI applications, security concerns dictate that keys be generated oncard. The private key should stay there, never read by any human. The public key can be released off-card to a PC or other device when the appropriate commands are issued. A hardware random-number generator must be accessible on-card by the CPU for this purpose. If only signing is needed, then the RNG does not need to be all that fast, because with each signing action only around 8 bytes of entropy is needed. During key generation, it is better to have a faster RNG; it is feasible that a cryptography-grade software PRNG be used, seeded with a hardware randomness source. It would be slow, but not overly so. According to local vendors, in practice the marginal cost of adding a hardware RNG is very low, essentially nil.

Access to random number generation hardware is either implemented via an I/O port read or as special memory whence a random byte can always be read. Usually it is as easy as using a single movx instruction (takes about 4 instruction cycles including setup). In our tests, the sampling of a random byte is implemented as a separate "black-box" routine in assembly that returns a value after about 10 instruction cycles, because we wish to account for slower hardware RNG implementations.

For signing, it is assumed that only the hash value of a message needs to be passed into a smart card. For key generation, the smart card needs to be able to save the key to EEPROM (flash memory) or spitted out of the card in blocks of an appropriate size. In general, the smart card is wired up to transmit data only in blocks of 128 or 256 bytes (at most) at a time, and the transfer rate is about 9600 baud, which makes for an effective bandwidth of at most $1 \mathrm{kB} / \mathrm{s}$. 